\title{
General Purpose Wireless Communication System Using Data Acquisition
}

\author{
Abdullah Eroglu, Bill Westrick \\ Indiana University-Purdue University Fort Wayne, Fort Wayne, USA \\ Email: eroglua@ipfw.edu
}

Received August 29, 2011; revised October 12, 2011; accepted November 10, 2011

\begin{abstract}
A sensor-enabled and RFID-integrated, Wireless Data Acquisition System (WDAS) that has similar functionality as multiple input multiple output (MIMO) systems for health-care applications has been developed, simulated and implemented. The system can accept various measurements via sensors placed on patients or elders and transmit information wirelessly to base station at a remote location. A microcontroller is used to control and process the signals submitted from sensor-enabled RFID devices using sequential logic. Processed signals are then sent to single receiver and transmitter pair instead of conventional MIMO antenna systems. This reduces the cost significantly in comparison to existing systems. It has been shown that the data transmitted from measurement locations can be acquired at the base station located 20 mile away. Data acquisition system (DAQ) is used to determine the measured value, apply the required statistics and display information to health-care professional at the base station. The system developed can be used for health-care applications including nursing homes in rural areas where access is difficult.
\end{abstract}

Keywords: WDAS; LabView; Multiple Input Multiple Output RFID; Sensor; Sequential Logic

\section{Introduction}

The recent developments in technology enabled professionals to use real time wireless data acquisition (WDAS) technology in several areas including structural health monitoring and security systems, manufacturing plants, and health-care applications [1-3]. One of the common problems in WDAS is the distance between receiver and transmitter to have high accuracy and low cost $[4,5]$. If multiple measurements are required, this problem then gets worse and transmission problem and system implementation difficulties arise. Multiple input and multiple output (MIMO) systems usually require several antennas at the transmitted and receiver locations. This adds another challenge to the system implementation by increasing the circuit complexity and its associated cost.

In this paper, sensor-enabled RFID device is used with a portable WDAS that can accept multiple measurements via sensors placed on patient or elder and transmit the measured signals wirelessly to base station which is located 20 mile away from the measurement point. System uses sequential logic with microcontroller and single RF transceiver system to reduce the cost and increase the communication distance. This eliminates the need to use conventional MIMO system where multiple antennas are needed at the input and output and hence reduces the cost significantly. Base station has RF receiver, receiver mi- crocontroller for post processing of the measured signals, and data acquisition system (DAS). DAS is equipped with a SC-2345 signal conditioner, 16 bit DAQ PAD 602E hardware, converter and interfacing circuits. DAQ system is used to determine the measured values, calculate of statistics to predict the behavior of the physical variables that are being measured, and display the results to health-care professionals. This system is general-purpose long-range low cost WADS that has same functionality as MIMO and can be used in applications including health-care, and structural health monitoring. Implementation of such as system with senor-enabled RFID device is illustrated in Figure 1.

\section{Proposed Architecture of WDAS}

The block diagram of the proposed MIMO WDAS is illustrated in Figure 1. System has seven different parts, which are labeled based on their operation. The first part of the system is composed of measurement instruments such as sensor-enabled RFID device and their corresponding interfacing circuits. Pre-transmission conditioning circuit constitutes the second part. The third and fourth parts consist of RF wireless transmitter and receiver antennas, respectively. The post receiver conditioning circuit is located in the fifth part. Signal conditioning takes place in the fifth part. The final stage is the 


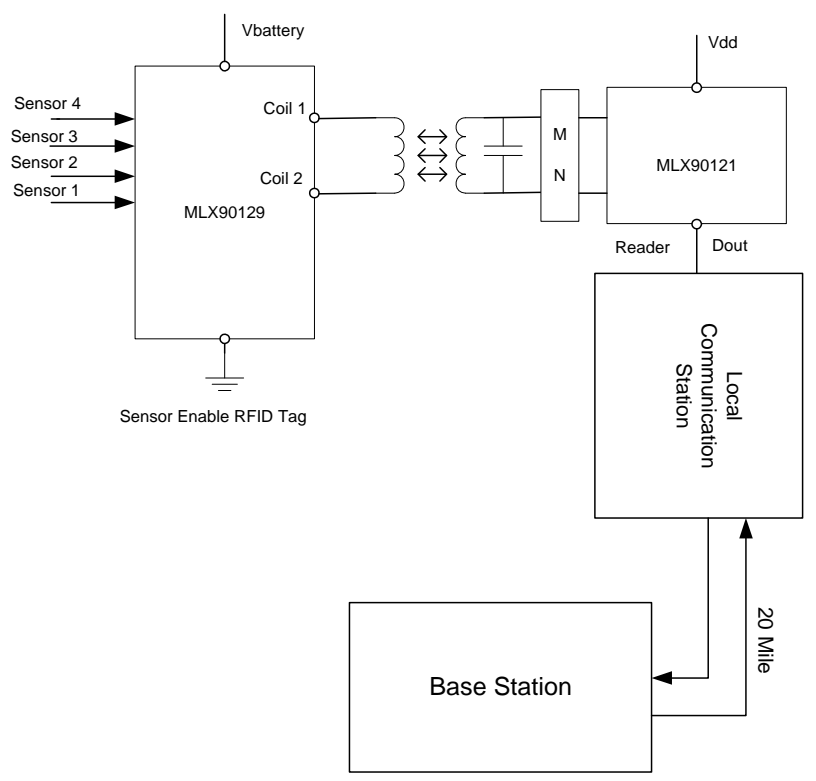

Figure 1. Implementation of sensor-enabled WDAS system.

computer where all the measured values are processed, and displayed via LabView program. The custom designed conditioning circuits are used to convert the analog signal from the RFID device into digital signal that will be fed to microcontroller. It is also used to encode signal that will be transmitted with the proper format. The post-receiver conditioning circuit reverses the operation of pre-transmission circuit and converts the transmitted signal back into an analog signal and normalizes it for SC-2345, signal conditioning unit. DAS unit, which has DAQ PAD 602E hardware with computer and LabView program processes the signals that will be displayed to the health-care professional.

Signal transmission is accomplished using digital format because digital signal transmission technology has high anti-jamming ability and reliability. RF transceiver pair ConnexLink (CL) 4790 [6] is used as a wireless transmission and reception module to increase the communication distance. Its frequency band changes between 902 to $928 \mathrm{MHz}$. It features Frequency Shift Keying (FSK) modulation and demodulation capability and it has transmission bit rates up to $115.2 \mathrm{kbps}$. Sensitivity of the module at full RF Data Rate is $-99 \mathrm{dBm}$. The information regarding to RF transceiver's data rate, power are provided in [6].

\section{Implementaion and Simulation}

System architecture that is proposed in Section 2 and shown in Figure 2 is implemented as a block diagram in Figure 3. It has mainly the transmitter section where all the measurements have been performed and signal transmitted and receiver section where all signals are captured, processed and displayed to the health-care pro-

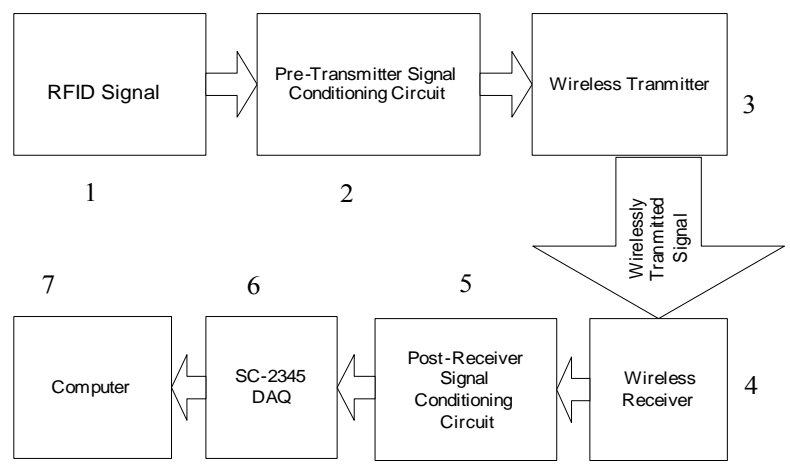

Figure 2. General block diagram of WDAS.

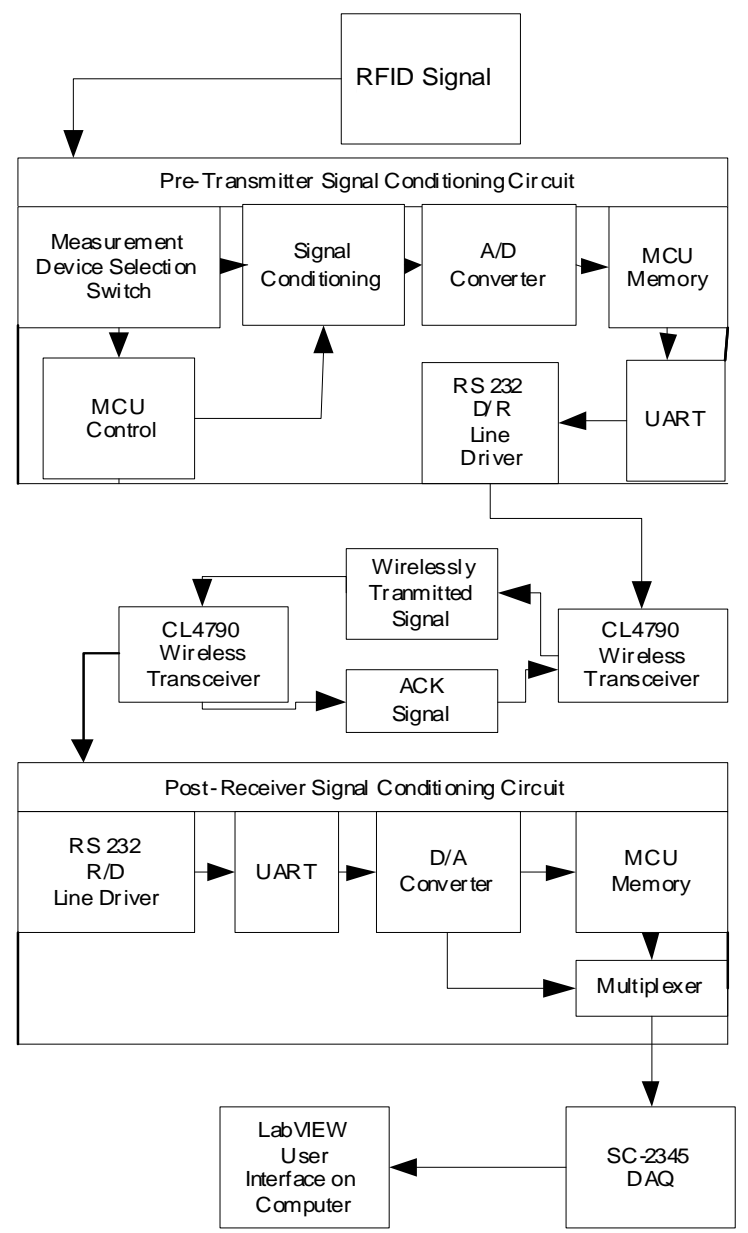

Figure 3. General block diagram of multiplexing circuit using the ConnexLink 4790.

fessional.

Single RF channel is used to transmit multiple data signals using sequential logic with this implementation method. Each sensor uses a separate circuit to measure and condition a signal in the range that is used by ADC. The measurement circuits are connected either directly to a multi-channel ADC, or to a single-channel ADC via a multiplexer. 
The transmission circuit is controlled by microcontroller C8051F340 that selects the signal to be processed by digital to analog converter (DAC). The microcontroller either cycles through each input channel, or through only the input channels selected by means of DIP switches. Once the conversion is completed, the digitized value is transferred to the microcontroller's memory. The address of the multiplexer selecting the signal is appended to the signal's value. This combined data packet is then loaded into a UART. Hence, data packet is converted into a bit stream by this way.

The next stage is RS-232 line driver, which converts the TTL bit stream into higher voltage RS-232 bit stream in order to communicate with the CL4790. There are two input signals that are sent to the MCU. There is 12-bit digital measurement signal and 6-bit acknowledgement and select signal. 12-bit digital measurement signal comes from one of the five measurement devices that had been polled by the MCU and the analog to digital converter.

6-bit acknowledgement and select signal come from the receiver. 6-bit acknowledgement and select signal are composed of 3-bit select signal and 3-bit acknowledgement signal. 12-bit measurement signal is sent to the MCU's universal asynchronous receiver/transmitter or UART. 6-bit acknowledgement signal is split between 3 separate 1-bit acknowledgement signals that are sent to an acknowledgement circuit and 3-bit select signal. 12bit output of the UART and 3-bit select signal are then sent for sampling. The sampling code uses the MCU clock to choose a single 12-bit measurement signal and appends 3-bit label to create 15-bit output that is sent to the RS-232 Line Driver and then to the transmitter. The sampling code also creates 3-bit select signal to be sent to MUX for measurement device selection.

The receiver circuit is controlled by the second microcontroller C8051F340. There are two input signals that are sent to the receiver MCU. These signals are 12-bit measurement signal from the transmitter and 3-bit select signal.

The 15-bit measurement signal from the transmitter is composed of 12-bit measurement signal from one of the measurement devices. 3-bit label designates the measurement device. 15-bit signal is first split into two signals, 3-bit label and 12-bit measurement signal. 12-bit signal is then sent via microcontroller's UART to digital to analog converter. MCP 4822 is selected as digital to analog converter. Timing Diagram for the DAC conversion process is shown in Figure 4.

After D/A conversion, the signal is sent for signal conditioning. 3-bit label is sent into two different segments of the program. It is first is sent to the SC-2345 signal conditioning box using 3-digital input ports for identification of the measurement signal. Acknowledgement signal is sent back to the transmitter to confirm the reception of the signal at the receiver end. 3-bit acknowledgement signal is then combined with the 3-bit select signal to form 6-bit signal that is sent to the UART at microcontroller and then to the RS-232 Line Driver.

The DAS receives two input signals: a 3-bit Label and a $5 \mathrm{~V}$ measurement signal. 3-bit signal is then sent into the digital input ports on the SC-2345 signal-conditioning box. $5 \mathrm{~V}$ measurement signal is sent to SC-2345 using $5 \mathrm{~V}$ analog input channel. The DAS software, LabView, uses label to identify the measurement device that the signal is coming from. The signal is then processed using the corresponding algorithm generated. The results are then displayed using LabView front panel.

The transmitter and the receiver side of WDAS is simulated using National Instruments Multisim V11 circuit simulator. The layout of the transmitter circuit that is simulated illustrated in Figure 5.

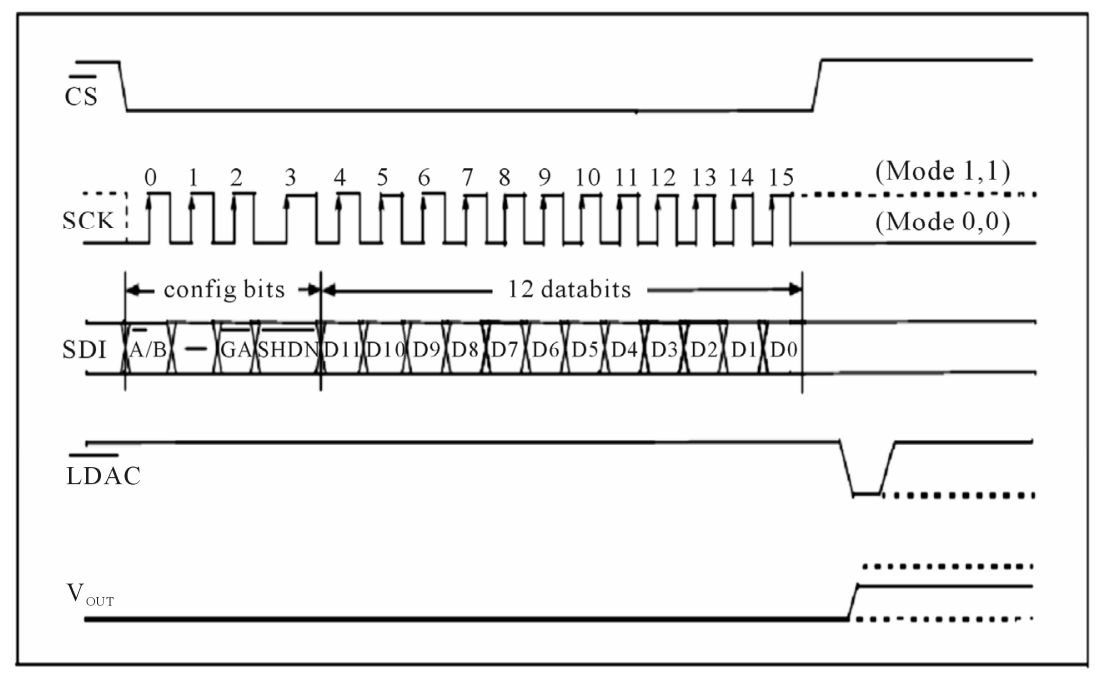

Figure 4. Timing diagram of MPC 4822. 


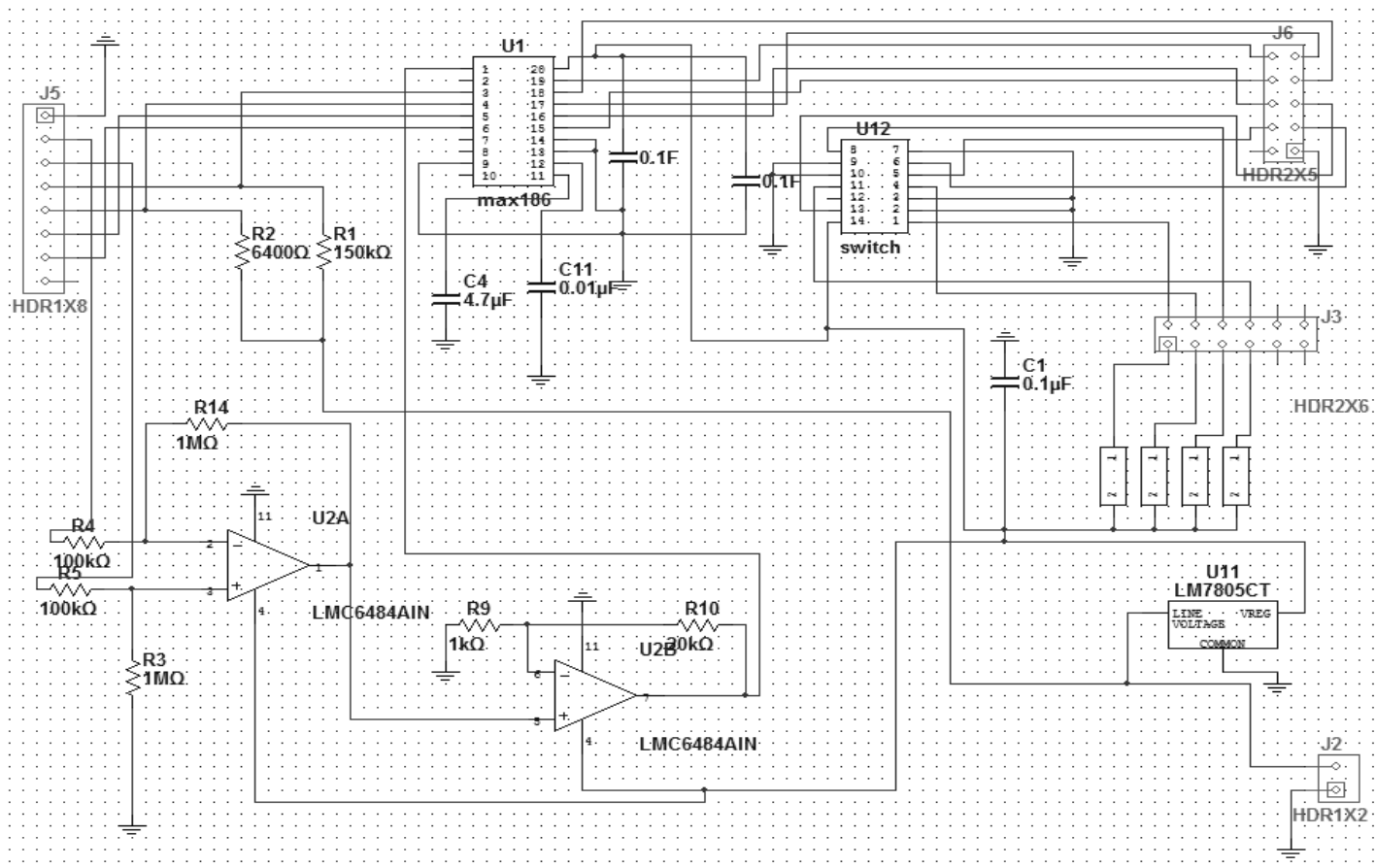

Figure 5. Layout of the simulated transmitter circuit for WDAS.

The RFID sensor circuit is also simulated for several different physical measurements. One of the input measurement devices is chosen to be a thermistor to measure temperature. The temperature that is measured by thermistor is calculated using a third order Steinhart-Hart equation as

$$
\frac{1}{T}=A+B \ln (R)+C(\ln (R))^{3}
$$

where $T$ is temperature in Kelvin, $R$ is the resistance in ohms, $A, B, C$ are Steinhart-Hart coefficients that depend on the type of the semiconductor material. The equation given in (1) can be simplified by setting $C=0$ and $B=1 / b$. The equation then simplifies to

$$
R=R_{0} \mathrm{e}^{\beta\left(\frac{1}{T}-\frac{1}{T_{0}}\right)}
$$

In Equation (2), $R_{0}$ is defined as base resistance $T_{0}$ is defined as base temperature. Beta is calculated from

$$
\beta=\ln \left(\frac{R}{R_{0}}\right)\left(\frac{T_{0} T}{T_{0}-T}\right)
$$

Taking the natural $\log$ of Equation (3) gives the following equation

$$
\ln (R)=\beta\left(\frac{1}{T}-\frac{1}{T_{0}}\right)+\ln \left(R_{0}\right)
$$

Plotting $\ln (R)$ versus $\left(1 / T-1 / T_{0}\right)$ gives a linear response with the gradient $\beta$ as shown in Figure 6. That can be used to extract the measured temperature versus resis- tance change in the termistor as depicted in Figure 7. The temperature measurement circuits that is used for simulation to measure temperature is given in Figure 8.

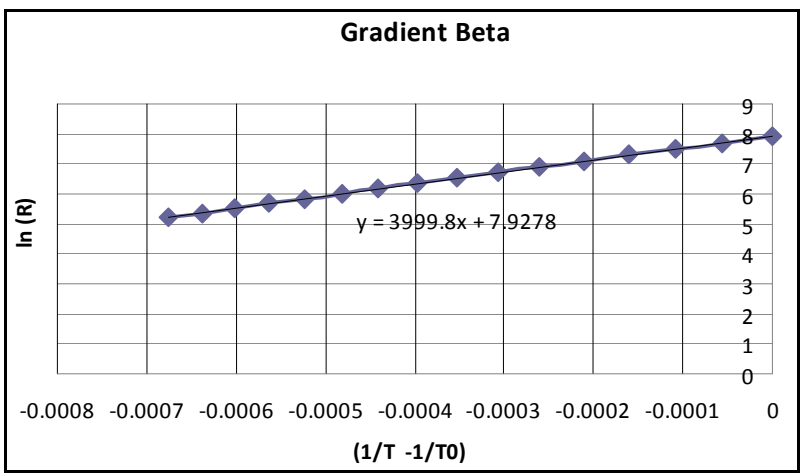

Figure 6. Plot to determine $\beta$ value for thermistor.

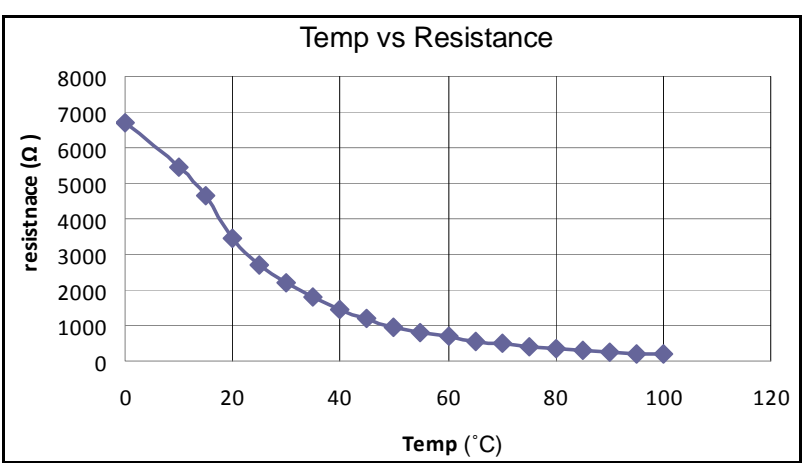

Figure 7. Simulated temperature measurement with thermsitor. 


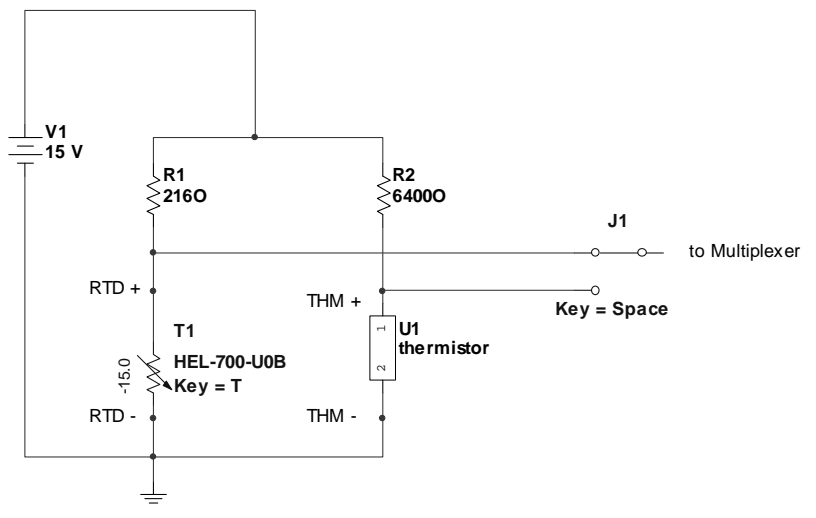

Figure 8. Simulated temperature input measurement circuit for WDAS.

The measurement circuit shown in Figure 8 is then integrated with the input interfacing circuit that is connected to transmitter. The simulated circuit consisting of measurement circuit is given in Figure 9. The simulated values closely matched with the measured values.

\section{Measurement Results}

WDAS for long-range application using sequential logic is implemented and measured. The test has been performed in an outdoor environement. The transmitter and receiver have been separated up to 20 mile. The base station where receiver and DAS with all the hardware and software are located inside a closed structure where power was available. The transmitter is located outside and power is supplied using a portable power supply module.
It has been observed that the transmission and reception was good when there were no obstacles such as trees and buildings between the receiver and transmitter. Better communication was possible with the replacement of longer range receiver and transmitter antenna system. The measurement results at the receiver and transmitter sides are shown in Figures 10 and 11, respectively.

The trace that is colored in blue in Figures $\mathbf{9}$ and $\mathbf{1 0}$ is the measurement signal. Figure $\mathbf{9}$ shows the transmitted measurement signal whereas Figure 10 shows the reconstructed signal at the base station. The signal that is colored in orange is the clock signal. As it is shown, WDAS was successfully implemented and used to transmit and receive the measurement signal. At the base station this acquired signal is then transmitted to DAS using LabView. The signal that is received is processed and the measurement values are displayed. This is illustrated in Figure 12.

\section{Conclusions}

In this paper, design, simulation and implementation of a long-range portable MIMO wireless data acquisition system using sequential logic, which can accept several different measurement signals from sensor enabled RFID device for health-care applications is presented. It has been shown that the method that is used is superior to the existing systems in terms of cost and implementation. This type of MIMO system requires only one set of receiver and transmitter antenna system using the proposed method. The DAS system equipped with LabView at the

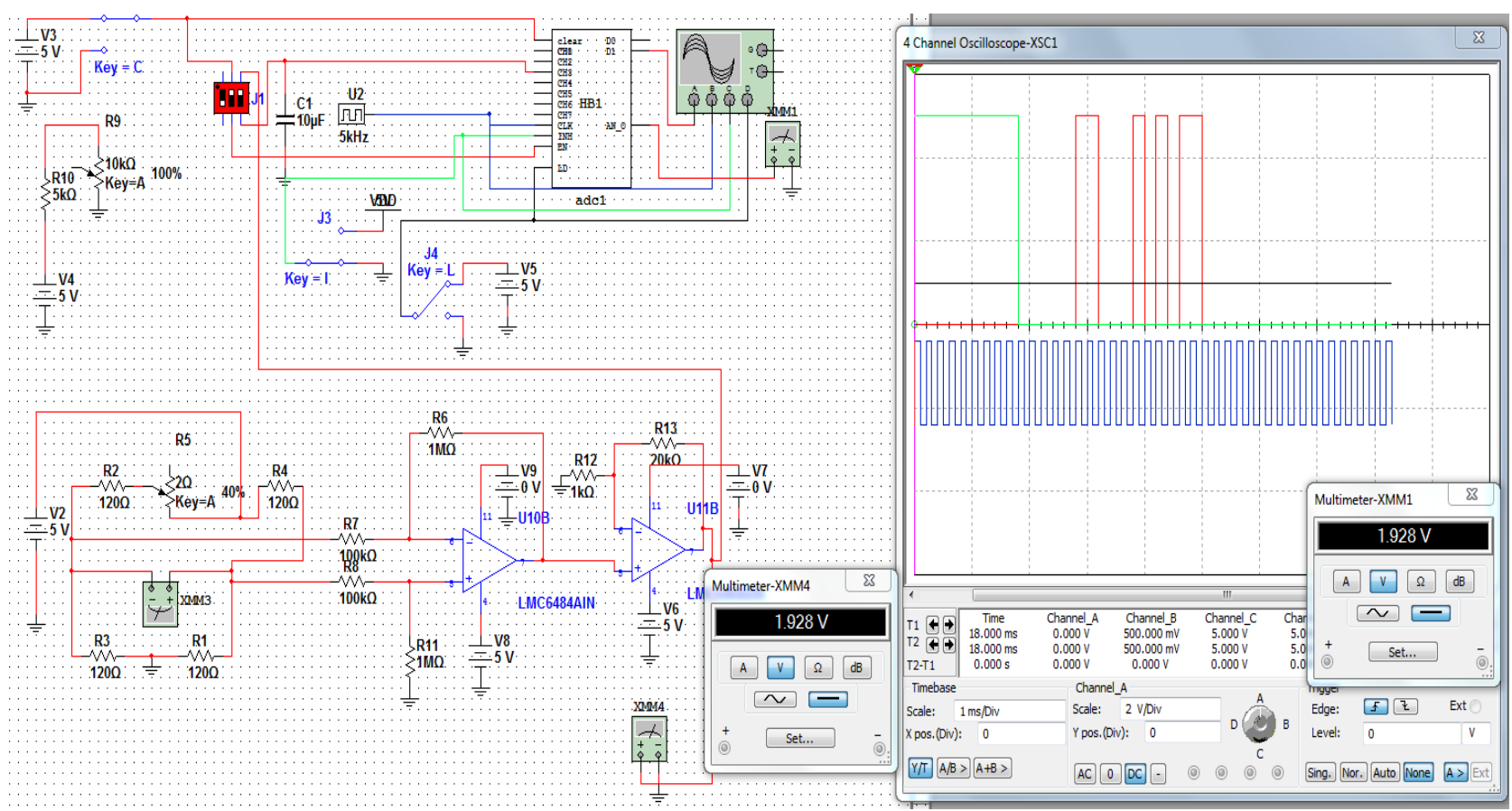

Figure 9. Simulated input interfacing circuit for WDAS. 


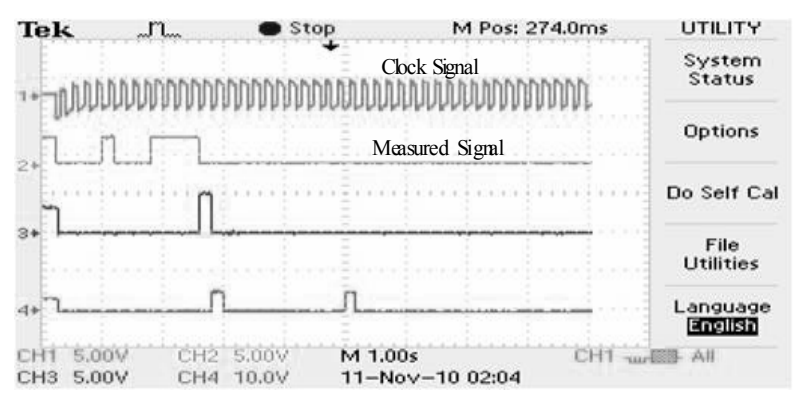

Figure 10. Measurement result at the receiver for WDAS.

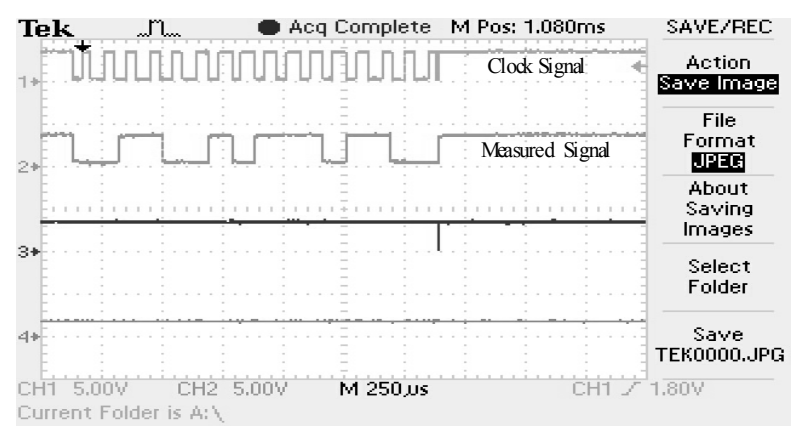

Figure 11. Measurement result at the transmitter for WDAS.

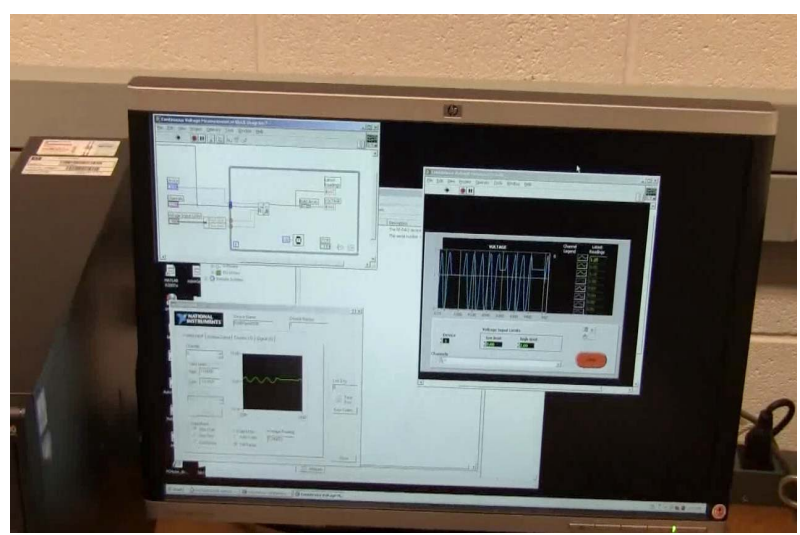

Figure 12. Labview display for WDAS. base station facilitates the processing of the data that is acquired and displays the final measured value. It is also capable of doing statistical analysis and can be used as a warning system to raise the flag when such as condition occurs. The system that is developed can be utilized in several other applications in addition to health-care applications such as structural health monitoring systems, and security systems.

\section{REFERENCES}

[1] K. Arshak, F. Adepoju and E. Jafer, "Simulation and Characterization of Wireless Data Acquisition RF Systems for Medical Diagnostic Application," Proceedings of IEEE Design and Diagnostics of Electronic Circuits and Systems (DDECS), Kraków, 11-13 April 2007, pp. 1-4. doi:10.1109/DDECS.2007.4295268

[2] K. Arshak and F. Adepoju, "Verification of Wireless Data Acquisition Radio Frequency System for Medical Telemetry Application," International Spring Seminar on Electronics Technology, Cluj-Napoca, 9-13 May 2007, pp. 439-444.

[3] A. Dinh, D. Teng, L. Chen, S. B. Ko, Y. Shi, J. Basran and V. Del Bello-Hass, "Data Acquisition System Using Six Degree-of-Freedom Inertia Sensor and Zigbee Wireless Link for Fall Detection and Prevention," Proceedings of IEEE Engineering in Medicine and Biology Society (EMBS), Vancouver, 20-24 August 2008, pp. 2353-2356.

[4] L. X. Wang, S. Wei and L. Chao, "Implementation of High Speed Real Time Data Acquisition and Transfer System," Proceedings of IEEE Industrial Electronics and Applications Conference (ICIEA), Xi'an, 25-27 May 2009, pp. 382-386.

[5] Z. Yu, J. Wu, M. Xie and Y. Kong, "Implementation of Distributed High Precision Real-Time Data Acquisition System," Proceedings of IEEE-NPSS Real Time Conference, Beijing, 10-15 May 2009, pp. 446-449. doi:10.1109/RTC.2009.5321600

[6] CL4790-1000-232, "ConnexLink-Wireless Cable Replacement System,” Datasheet. 